\title{
An Interesting Presentation of Severe Bicytopenia in A Case of SLE
}

\author{
M. Aswin Manikandan*, A. Josephine, Vindu Srivastava and S. Mary Lilly \\ Department of Pathology, Sree Balaji Medical College and Hospital
}

\section{ABSTRACT}

We present a case of 25-year-old female who was brought to the hospital for complaints of generalised weakness, fever, and history ofmelena and haematuria. Following admission complete blood count and peripheral smear was asked; Complete blood count (CBC) findings were haemoglobin $4.2 \mathrm{gm} / \mathrm{dl}$, total WBCcount was 14,990, platelet count 7000, reticulocyte count 4\%, NRBCswere $15 / 100$ WBCs. Peripheral Smear showed fragmented RBCs, polychromatophilsmicrospherocytes and multiple foci of autoagglutinationsuggestive of autoimmune haemolytic anaemia. Pertaining to these findings immune workup was done for this patient; coombs test was negative for this patient, but ANA was positive. This identification proved valuable to the patient as; administration of corticosteroids helped to prevent haemolytic transfusion reactions and improved the patient's haemoglobin and platelet count.

\section{Keywords: ANA, Anti Nuclear Antibody}

\section{Introduction}

Immune haemolytic anaemia is caused by binding of antibodies to red cell membranes leading to their premature destruction. Autoimmune haemolytic anaemia (AIHA) is a rare disease with an incidence of only 1-3 per $1,00,000$ people per year. AIHA occurs in 5-10\% of Systemic lupus erythematosus (SLE) patients ${ }^{[1]}$. Thrombocytopenia is a frequent presentation in case of a SLE patient. Identification of immune nature of the haemolytic anaemia is important as it can prevent the adverse effect of haemolytic transfusion reaction due to preformed antibodies.

\section{Case Report}

A 25-year-old female was brought to the hospital for complaints of generalised weakness, decreased appetite, headache, and fever. She had frontal headache and fever for 3 days. On obtaining history, it was noted that, she had history of nausea for past 10 days and lower abdominal pain for past 3 days, history of melena and haematuria were also noted. There was decrease in level of consciousness with a Glasgow coma scale (GCS) score of 3. Following admission peripheral smear was asked; the findings were haemoglobin $4.2 \mathrm{gm} / \mathrm{dl}$, total WBC (white blood cell) count 14,990, platelet count 7000, reticulocyte count $4 \%$, NRBCs(nucleated red blood cell) 15/100 WBCs (Figure 2), serum LDH (Lactate dehydrogenase) - 1555 IU/L. Coombs test was negative, but ANA (Anti-nuclear antibody) was positive.

Peripheral smear showed predominantly microcytic hypochromic cells with normocytic normochromic cells showing moderate anisopoikilocytosis comprising of schistocytes, polychromatophils, microspherocytes. Autoagglutination was noted. Nucleated RBCs were 15/100 WBCs. Total count was increased with neutrophil showing shift to left up to myelocyte. Platelet count was severely reduced. no haem parasites were seen in the peripheral smear.

\section{Discussion}

SLE can occur in any age group but it is more prevalent in women of childbearing age group of 15-44 years. ${ }^{[2]}$

Presence of fragmented RBCs (Figure:1) and thrombocytopenia in peripheral smear were reported to the clinician immediately as plasma transfusion can alleviate disseminated intravascular coagulation. On further study, other findings like presence of micro spherocytes (Figure: 3 ), autoagglutination and presence of polychromatophils (Figure:1) suggested autoimmune haemolytic anaemia

Immune haemolytic anaemia may be the first manifestation of SLE before other symptoms, and other organ systems are affected. Anaemia in SLE may be due to multiple causes, they are anaemia of chronic disease, blood loss (gastrointestinal loss, menorrhagia), nutritional deficiency, immune mediated, myelofibrosis and treatment induced. Immune mediated haemolysis is due to anti-erythrocyte antibodies acting on the red cell membrane and is usually of IgG class antibodies.

In a study conducted by A. Kalfa they have shown that, $3-11 \%$ of cases included in their study had Direct 
antiglobulin test (DAT) negative warm-AIHA. Coombs test has been found negative in few cases of autoimmune haemolytic anaemia and the reasons were due to presence of low levels of $\mathrm{IgG}$ sensitization which was not detected by antiglobulin reagent, low affinity IgG antibodies which are removed by preparatory washes during the test, and when red cell sensitization occurs by $\operatorname{IgA}$ or IgM alone. ${ }^{[3,4]}$

This patient also showed increased serum LDH- 1555 $\mathrm{IU} / \mathrm{L}$ which is also a sign of immune haemolytic anaemia. ${ }^{[5]}$

Increase in leukocyte count with leucoerythroblastic picture along with thrombocytopenia can also be a presenting feature in SLE. ${ }^{6]}$ This is seen in severe cases of autoimmune haemolytic anaemia.

The presentation of severe autoimmune haemolytic anaemia and severe thrombocytopenia mimics Evan's syndrome, but in our case; the nature of antibody affecting the platelets were not identified. ${ }^{[7]}$

A differential diagnosis of haemolytic uremic syndrome was considered but the absence of signs of renal failure or neurological symptoms excluded this diagnosis. Also, a differential diagnosis of sickle cell anaemia was considered owing to younger age of the patient and haemolytic blood picture. Sickling test was done and turned out to be negative.

\section{Conclusion}

Prompt identification of immune haemolytic blood picture is important as it can influence the treatment strategy and prognosis of the patient. In this patient, corticosteroids were given before the transfusion procedure, which prevented the haemolysis and facilitated the increase in haemoglobin and platelet count.

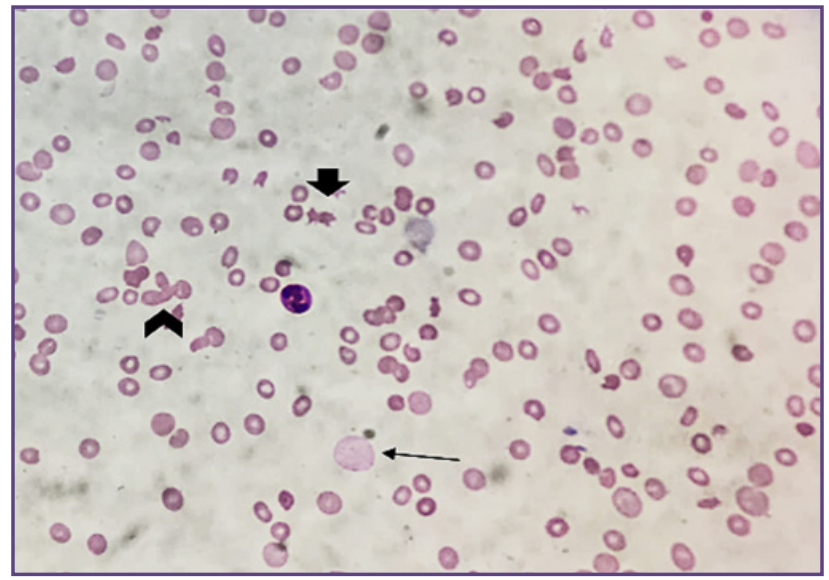

Fig. 1: smear shows anisopoikilocytosis with multiple fragmented RBCs(short arrow), polychromatophils(long arrow) and foci of autoagglutination (arrowhead) (x400, Leishman stain).

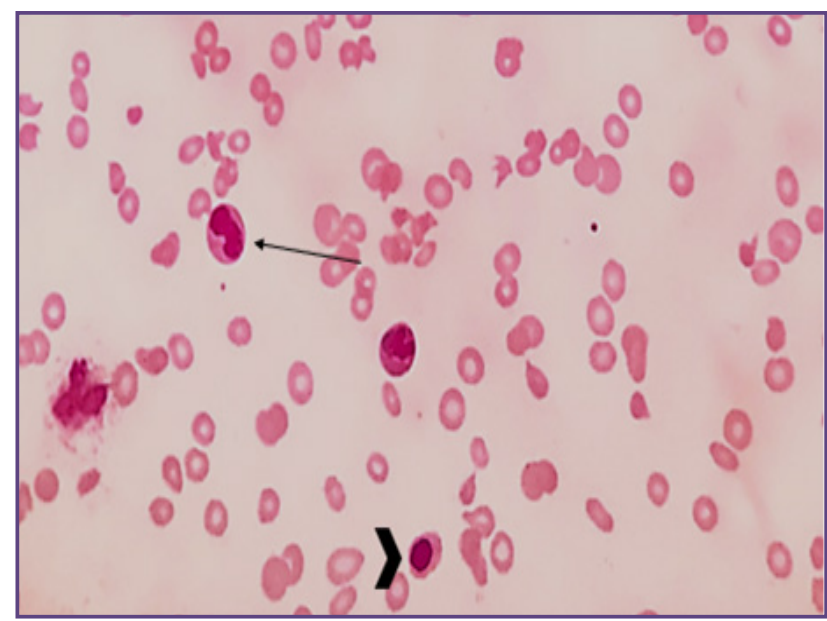

Fig 2: show shift to left with metamyelocyte (long arrow) and has nucleated RBC(arrowhead) (x400, Leishman stain).

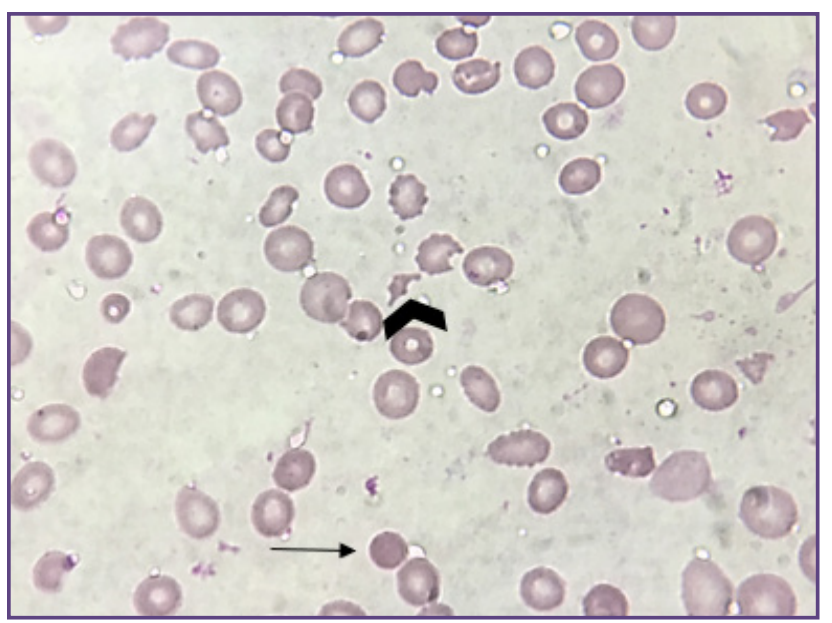

Fig. 3: Fragmented RBCs (arrowhead) and micro spherocyte (long arrow) (x1000; oil immersion, Leishman stain). 


\section{References}

1. Kalfa TA. Warm antibody autoimmune hemolytic anemia. Hematology Am Soc Hematol Educ Program. 2016;2016(1):690-697. doi:10.1182/ asheducation-2016.1.690

2. Dall'Era M. Systemic lupus erythematosus. In: Imboden JB, Hellman DB, Stone JH. (Eds). Current Rheumatology Diagnosis and Treatment. 3rd ed. New York, NY:McGrawHill; 2013.

3. Segel GB, Lichtman MA. Direct antiglobulin ("Coombs") test-negative autoimmune hemolytic anemia: a review. Blood Cells Mol Dis. 2014 Apr;52(4):152-60. doi: 10.1016/j. bcmd.2013.12.003

4. Kalfa TA. Warm antibody autoimmune hemolytic anemia. Hematology Am Soc Hematol Educ Program. 2016;2016(1):690-697. doi:10.1182/asheducation-2016.1.69

5. Kato GJ, McGowan V, Machado RF, et al. Lactate dehydrogenase as a biomarker of hemolysis-associated nitric oxide resistance, priapism, leg ulceration, pulmonary hypertension, and death in patients with sickle cell disease. Blood. 2006;107(6):2279-2285. doi:10.1182/ blood-2005-06-2373

6. Disorder of Red Blood Cells: Anemias,Editor(s): Faramarz Naeim, P. Nagesh Rao, Wayne W. Grody, Hematopathology, Academic Press, 2008, Pages 529-565

7. Shaikh H, Mewawalla P. Evans Syndrome. [Updated 2020 Jun 23]. In: StatPearls [Internet]. Treasure Island (FL): StatPearls Publishing; 2020 Jan.

*Corresponding author:

Dr. M. Aswin Manikandan, A25A, Desh Apartment, urapakkam, Chennai-603210,

Phone: +91 8220153399

Email: aswinm1987@outlook.com

Financial or other Competing Interests: None.

Date of Submission : 07/12/2020

Date of Revision $\quad: 05 / 01 / 2021$

Date of Acceptance : 14/01/2021

Date of Publication : 30/01/2021 